\title{
Anticholinergic Drugs and Their Effects on Delirium and Mortality in the Elderly
}

\author{
M.J. Luukkanen ${ }^{a, c} \quad$ J. Uusvaara ${ }^{a} \quad$ J.V. Laurila ${ }^{b}$ T.E. Strandberg ${ }^{d}$ \\ M.M. Raivio ${ }^{a}$ e R.S. Tilvis ${ }^{b}$ K.H. Pitkäläa \\ a Unit of General Practice and ${ }^{b}$ Department of General Internal Medicine and Geriatrics, \\ Helsinki University Central Hospital, Helsinki, 'Sipoo Community Health Center, Sipoo, \\ ${ }^{d}$ Institute of Health Sciences/Geriatrics, University of Oulu, and Unit of General Practice, \\ Oulu University Hospital, Oulu, and ' Social Insurance Institution of Finland and University \\ of Helsinki, Helsinki, Finland
}

\section{Key Words}

Anticholinergic effects $\cdot$ Comorbidities $\cdot$ Delirium $\cdot$ Dementia $\cdot$ Elderly patients

\begin{abstract}
Aim: To investigate the use of drugs with anticholinergic properties (DAPs) and their associations with delirium and mortality among elderly patients with comorbidities. Methods: 425 patients ( $\geq 70$ years of age) in geriatric wards and nursing homes were assessed. The use of DAPs was retrieved from their medical records. Delirium was diagnosed according to the DSM-IV criteria. Results: Of the 341 patients (80.2\%) treated with multiple DAPs ( $\geq 2), 92$ patients $(27.0 \%)$ suffered from delirium, whereas 14 of 84 patients (16.7\%) without DAP treatment had delirium $(p=0.050)$. In a logistic regression analysis with age, gender, and Charlson Comorbidity Index as covariates, DAP treatment did not predict delirium (odds ratio $1.67,95 \%$ confidence interval 0.87-3.21). The 2-year mortality was $49.3 \%(n=168)$ in DAP users and $35.7 \%(n=30)$ in non-users, respectively ( $p=0.026)$. In the Cox proportional hazard model adjusted for age, gender, and comorbidity, DAPs did not predict mortality (hazard ratio 1.12, 95\% confidence interval $0.75-$ 1.68). Conclusion: The use of DAPs is very frequent among frail inpatients with comorbidities, but their use has no independent prognostic significance.

Copyright $\odot 2011$ S. Karger AG, Basel
\end{abstract}




\section{Introduction}

Delirium is a common clinical problem in elderly patients in hospitals and in nursing home residents. Its prevalence varies from 1 to $56 \%$, depending on the population studied [1] and the diagnostic tools and criteria chosen [2]. The etiology of geriatric delirium is multifactorial. It consists of predisposing factors adding to patients' baseline vulnerability (e.g. dementia) and precipitating (noxious) factors (e.g. major surgery), which may lead to delirium in complex interaction [3,4]. Delirium prolongs hospital stay [5], increases the need of care, and is associated with increased mortality [6].

While cognitive impairment is one of the major factors predisposing to delirium [7], adverse effects of drugs may be an important precipitating factor $[4,8]$. Experimental and clinical studies have shown that in a vulnerable person delirium can be induced by treatment with scopolamine, an anticholinergic drug, and it can be reversed with physostigmine, a potent cholinomimetic drug [9]. It has been demonstrated that regular use of drugs with anticholinergic properties (DAPs) can cause cognitive decline in older individuals [10-13].

Characteristic symptoms of delirium are supposed to be mediated by acetylcholine deficiency and dopamine excess, both in absolute amounts and/or relative to each other [14, 15]. Many commonly prescribed as well as over-the-counter drugs have anticholinergic properties of varying degree. It is thus of clinical interest to clarify whether the use of DAPs is associated with delirium and poor outcome in elderly patients, especially in those with dementia.

This study has three aims: (1) to investigate the use of DAPs in elderly patients with comorbidities in acute geriatric wards and nursing home residents; (2) to clarify the relationship between the use of DAPs and delirium, and (3) to study the prognostic significance of DAPs regarding mortality.

\section{Material and Methods}

This study was originally designed to assess the phenomenology of delirium [16]. The database included detailed assessments of all eligible patients in 7 acute wards of 2 geriatric hospitals, and of residents in 13 wards of 7 nursing homes in Helsinki, Finland. Representative, average-sized nursing homes were chosen for the study. After a complete description of the study had been provided, all individuals who had given informed consent were included in the study, and in those with a poor capacity for judgment, informed consent was obtained from the patients' closest proxy. The only exclusion criteria were age $<70$ years and/or coma.

All the patients in geriatric wards and the residents in nursing homes underwent thorough assessments to detect delirium and preexisting dementia. The study subjects were assessed using an extensive questionnaire including mental and cognitive tests, such as the Mini-Mental State Examination (MMSE), a 30-point test assessing global cognitive function [17]; Digit Span, a test of working memory and attention, in which the patient repeats a series of random digits forward and backward [18]; the Clinical Dementia Rating (CDR) scale, a 5-point scale in which a value of 0 denotes no cognitive impairment and the remaining 4 categories indicate various stages of dementia [19]; and the proverb part (testing abstract thinking and judgment) of the Wechsler Adult Intelligence Scale, the most commonly used intelligence scale for adults [20]. The assessment also included background information on the patient, such as demographic variables, diagnoses, and alcohol and tobacco use. All available medical records and nurses' notes both in the acute geriatric wards and in the nursing homes were reviewed by one of two skilled and experienced geriatricians (J.V.L. and K.H.P.) to obtain as much information as possible on medical history, acute medical prob- 
Table 1. DAPs according to our previous study [27] and further relevant literature [10, 23, 24, 25, 26, 28]

High anticholinergic properties

Amitriptyline [10, 25, 26, 27]

Clidinium [26, 27]

Emepronium [27]

Hydroxyzine [10, 25, 27, 28]

Levomepromazine [10, 26, 27]

Orphenadrine [27]

Oxybutynine [10, 25, 27, 28]

Periciazine [27]

Detectable anticholinergic properties

Alprazolam [10, 27]

Biperidine [25, 27]

Captopril [25, 27]

Chlorpromazine [10, 27]

Clomipramine [10, 25, 27]

Codeine [10, 25, 27]

Corticosteroids [25, 27]

Diazepam [27, 28]

Digoxin [10, 25, 27]

Doxepine [25, 26, 27, 28]

Dipyridamole [25, 27, 28]
Detectable anticholinergic properties

Disopyramide [25, 26, 27]

Flupentixole [26, 27]

Furosemide [10, 27]

Ipratropium bromide [25, 27]

Isosorbide mono-/dinitrate $[25,27]$

Metoclopramide [27, 28]

Nifedipine $[25,27]$

Olanzapine [27]

Perphenazine [26, 27]

Pilocarpine [24]

Prochlorperazine [27]

Quinidine [25, 27]

Ranitidine [25, 27]

Theophylline [10, 25, 27]

Thioridazine [25, 27]

Tolterodine [27]

Triamterene [27]

Tramadol [27]

Trimipramine [10, 25, 27]

Warfarin [25, 27]

lems, current diagnoses, and medication use, as well as to acquire information on symptoms and signs prior to the interview. Delirium was defined using the DSM-IV [21]. The criteria for delirium according to the DSM-IV were operationalized to simple yes/no questions and included in a questionnaire.

To enhance the accuracy of the patients' preexisting dementia diagnoses we re-evaluated them and based the dementia diagnoses in this study on global judgment (definite or probable) of three geriatricians (J.V.L., K.H.P., and T.E.S.) with the aid of the following information: (1) existing dementia diagnoses were evaluated; (2) the CDR scale [19] and the operationalized DSM-IV criteria for dementia served to support the diagnoses; (3) nurses and/ or caregivers were interviewed about the earlier cognitive performance and physical functioning of the patient; (4) results of brain computerized tomography/magnetic resonance imaging (36\% of the patients), electroencephalography (3\% of the patients), and the previous MMSE scores (32.5\% of the patients) were registered if available. All medical diagnoses were retrieved from the patients' records and confirmed by the study geriatricians. Comorbidity was computed for each resident using the Charlson Comorbidity Index [22], a weighted index that takes into account the number and the seriousness of a resident's comorbid diseases. It has been shown to be a valid measure of prognosis in older people [22].

Dependence on caregivers in activities of daily living was assessed according to the CDR scale item 'personal care' [19]: CDR class 1 or higher ('requiring at least prompting or assistance in dressing, hygiene, managing personal effects, or requiring much help with personal care, often involving incontinence') was defined as activities of daily living dependence.

Every medication of each patient was retrieved from medical records. We assessed the participants' use of DAPs as follows: they were listed according to previous scientific literature [10, 23-27] (table 1). Only the DAPs used in a regular daily sequence were registered. In order to confirm a significant anticholinergic effect, participants were divided into two groups according to their DAP use: subjects receiving $\geq 2$ DAPs and $<2$ DAPs. Mortality data were retrieved from central registers, and the follow-up was complete for $100 \%$ of the 
Table 2. Demographic characteristics of the study population divided by the use of DAPs ( $n=425)$

\begin{tabular}{lllc}
\hline & $\begin{array}{l}\text { DAP user }(\geq 2) \\
(\mathrm{n}=341)\end{array}$ & $\begin{array}{l}\text { DAP user }(<2) \\
(\mathrm{n}=84)\end{array}$ & p value $^{1}$ \\
\hline Mean age, years (SD) & $86.7(6.8)$ & $83.7(7.2)$ & $<0.001$ \\
Female, \% & 83.0 & 76.2 & 0.15 \\
Primary school or less, \% & 52.4 & 52.2 & 0.99 \\
Widowed, \% & 56.1 & 46.8 & 0.14 \\
Dependent in activities of daily living, \% & 74.9 & 83.1 & 0.11 \\
Mean MMSE (SD) & $13.3(7.9)$ & $11.3(7.6)$ & 0.045 \\
Mean number of medications (SD) & $8.9(3.0)$ & $6.1(3.1)$ & $<0.001$ \\
Mean Charlson Comorbidity Index (SD) & $2.4(1.6)$ & $1.5(1.2)$ & $<0.001$ \\
Dementia, \% & 57.2 & 71.4 & 0.017 \\
Delirium by DSM-IV, \% & 27.0 & 16.7 & 0.050 \\
Residence at the time of assessment, \% & & & \\
$\quad$ Acute geriatric ward (n = 230) & 56.9 & 42.9 & 0.021 \\
$\quad$ Nursing home (n= 195) & 43.1 & 57.1 & \\
2-year mortality, \% & 49.3 & 35.7 & 0.026 \\
\hline
\end{tabular}

${ }^{1}$ Differences between the groups were tested by $\chi^{2}$ test for categorical variables and by Mann-Whitney $\mathrm{U}$ test for non-normally distributed continuous variables. ${ }^{2}$ Based on a CDR 'personal care' $>1$ [19].

patients. The study was approved by the local ethics committees (the Ethics Committee of the Department of Medicine, Helsinki University Hospital, and the Helsinki City Ethics Committee).

\section{Statistical Analysis}

Differences between users and non-users of DAPs were compared using the $\chi^{2}$ test for categorical variables and the Mann-Whitney $U$ test for continuous variables. We used logistic regression [odds ratios (OR) with 95\% confidence intervals (CI)] analysis to test the independent association of the use of DAPs with delirium. Age, gender and Charlson Comorbidity Index were used as covariates. Cox proportional hazards analysis was used to model the prognostic significance of the use of DAPs on the risk of mortality [hazard ratio (HR) with 95\% CI]. The model was adjusted for age, gender, and Charlson Comorbidity Index. All data were analyzed using the NCSS statistical program for Windows (NCSS, Kaysville, Utah, USA). Results were considered statistically significant at a level of $\mathrm{p} \leq 0.05$.

\section{Results}

Among the 425 patients (women 81.6\%, $\mathrm{n}=347$ ), 230 were treated in acute geriatric wards and 195 were nursing home residents. The mean age of the study participants was 86.1 years [standard deviation (SD) 7.0; table 2].

Among all patients, $80.2 \%(\mathrm{n}=341)$ used at least 2 DAPs daily. The most frequently used DAPs were furosemide, isosorbide nitrate, digoxin, tramadol, dipyridamole, warfarin, triamterene, and prednisone (table 3). DAP users were older, had a higher number of comorbidities and consumed more drugs. Among all DAP users, 27.0\% $(n=92)$ were suffering from delirium at the time of the interview compared with $16.7 \%(n=14)$ of the non-users $(p=$ 0.050). In a logistic regression analysis with age, gender, and Charlson Comorbidity Index as covariates, the use of DAPs did not predict delirium (OR 1.67, 95\% CI 0.87-3.21). Among pa- 
Table 3. The 24 most frequently used drugs and numbers of users

\begin{tabular}{lrlr}
\hline Furosemide & 152 & Amitriptyline & 13 \\
Isosorbide nitrate & 115 & Codeine & 12 \\
Digoxin & 101 & Theophylline & 10 \\
Tramadol & 53 & Nifedipine & 10 \\
Dipyridamole & 34 & Tolterodine & 9 \\
Warfarin & 31 & Biperidine & 9 \\
Triamterene & 22 & Pilocarpine & 8 \\
Prednisone & 22 & Diazepam & 8 \\
Hydroxyzine & 16 & Alprazolam & 7 \\
Oxybutynine & 15 & Doxepine & 7 \\
Periciazine & 14 & Captopril & 7 \\
Ipratropium bromide & 14 & Metoclopramide & 6 \\
\hline
\end{tabular}

tients with prior dementia $(\mathrm{n}=255)$, delirium was found in $28.7 \%(\mathrm{n}=56)$ of DAP users and $16.7 \%(\mathrm{n}=10)$ of non-users $(\mathrm{p}=0.062)$.

Of the whole study population, $46.6 \%(\mathrm{n}=198)$ died within 2 years. The all-cause mortality was $49.3 \%(\mathrm{n}=168)$ in DAP users and $35.7 \%(\mathrm{n}=30)$ in non-users $(\mathrm{p}=0.026$; table 2$)$. In the Cox proportional hazard model, Charlson Comorbidity Index (HR 1.18, 95\% CI 1.081.29; $\mathrm{p}<0.001$ ), age (HR 1.06/year, 95\% CI 1.04-1.08; $\mathrm{p}<0.001$ ) and male gender (HR 1.55, 95\% CI 1.09-2.20; $\mathrm{p}=0.014$ ) were independently associated with mortality, whilst the use of DAPs was no longer an independent predictor (HR 1.12, 95\% CI 0.75-1.68; $\mathrm{p}=0.56$ ).

Among dementia patients, the mortality rates were $50.8 \%(\mathrm{n}=99)$ and $31.7 \%(\mathrm{n}=19)$ among DAP users and non-users, respectively $(\mathrm{p}=0.009)$. In patients with dementia, the use of DAPs did not predict mortality in the Cox proportional hazard model with age, gender, and Charlson Comorbidity Index as covariates (HR 1.41, 95\% CI 0.85-2.34).

The use of DAPs did not emerge as a significant prognostic factor in any subgroup analysis (patients admitted to acute geriatric wards, nursing home residents, persons with or without dementia, and patients $>85$ or $<85$ years of age). We also tested whether a cutoff point of $>3$ DAPs would strengthen the association: the 2-year mortality was 58 and $43 \%$ $(\mathrm{p}=0.006)$, and the delirium prevalence was 25 and $25 \%(\mathrm{p}=0.94)$ among users ( $>3$ DAPs $)$ and non-users ( $<3 \mathrm{DAPs})$, respectively.

\section{Discussion}

The use of multiple DAPs was very frequent in this elderly, frail population since we included all drugs defined as having anticholinergic properties in our list. Bivariate analyses suggested that the use of DAPs increased the risk for both delirium and mortality. However, in multivariate analyses, the associations were no longer significant.

It is important to recognize that many drugs in daily long-term use have anticholinergic properties and that the use of DAPs cannot be fully avoided. In fact, over $80 \%$ of the patients in our study were using multiple DAPs as part of their everyday medication. Many of these drugs are essential for cardiovascular patients, drugs like furosemide, isosorbide nitrate, digoxin, triamterene or warfarin cannot be simply withdrawn or replaced. In another study by our group, prednisone and tramadol were assessed to be the most common precipitating drugs for delirium [4], and these drugs are also often required. However, there were also many DAPs, such as hydroxyzine, oxybutynine, doxepine, diazepam and metoclopramide, which are considered inappropriate for older people [28]. 
DAP users were older and had more often comorbid disease, and they used more drugs than the non-users. Therefore, the higher prevalence of delirium and the worse prognosis among the DAP users compared with the non-users was expected. However, we failed to show an independent prognostic significance for DAP use in this population. For several reasons, the negative results should be interpreted with caution. Firstly, almost all subjects used at least 1 DAP and neither the short-term nor the long-term anticholinergic effect could be quantified in this setting. Secondly, DAPs are only one of the precipitating factors for delirium $[4,8]$ and their influence may be masked by other triggers, such as infections and metabolic disorders. Due to the large number of predisposing and precipitating factors for delirium [4], it is challenging to show an independent role for any single factor, for example DAPs, in cross-sectional studies. Thirdly, the statistical power of our study may not be sufficient to show differences between the groups, although this is one of the largest studies conducted on this topic. With a 5\% type I error and $80 \%$ power, approximately 288 patients in each group would have been needed to show a $10 \%$ difference.

Interestingly, the use of DAPs was more prevalent among patients without dementia compared to those with dementia. Therefore, the MMSE scores were also somewhat higher among DAP users. A large proportion of the patients with dementia resided in a nursing home, where physicians often tend to reduce DAPs because of hypotension, terminal stage, or dementia. We also know that doctors in these settings tend to avoid prescribing DAPs to patients with dementia in the first place. Therefore, it is notable that our sample included patients both in acute geriatric wards (prevalence of delirium 35\% [2]) as well as in nursing homes (prevalence of delirium 14\%). On the other hand, nursing home residents had a higher prevalence of dementia and, therefore, were more vulnerable to DAPs. In Finland, most of the acute care of nursing home residents takes place in nursing homes; thus, a heterogeneous patient cohort could be studied.

Our results do not confirm the results of a previous study suggesting an association between DAPs and excess mortality [29]. However, that study involved a highly selected population of hip fracture patients, and its results cannot be compared with our cohort or a general older population.

The strengths of our study include the following issues: the study population represented the clinical reality well and included a fairly large number of patients with delirium. The data were carefully collected, including the prompt documentation of confirmed diagnoses and medications used. The procedures to diagnose dementia and delirium were appropriate, and the 2-year follow-up was complete for $100 \%$ of the patients.

A limitation of our study is its cross-sectional nature concerning the use of DAPs. Because it was not randomized according to the use of DAPs, all confounding factors could not be controlled for. DAPs may also vary in their anticholinergic properties, and individual responses may vary, too [30]. These could not be measured in our study; however, their effect was minimized by setting the cutoff point to $\geq 2$ DAPs to ensure a significant anticholinergic effect. So far, there is no international consensus on the list of DAPs and the levels of their anticholinergic effect, especially among elderly vulnerable patients [13, 27, 31]. The list of DAPs varies considerably among countries. We used a list of DAPs which we have previously shown to be associated with cognitive decline among older persons [27].

While we did not find a correlation between increased mortality or increased incidence of delirium and DAP treatment, we think that further studies on other potential adverse effects as well as the overall effect of DAPs on older patients are required. These medications should be used with caution and at a minimum dosage, especially in patients with comorbidities or dementia. 


\section{References}

1 Fong TG, Tulebaev SR, Inouye SK: Delirium in elderly adults: diagnosis, prevention and treatment. Nat Rev Neurol 2009;5:210-220.

-2 Laurila JV, Pitkala KH, Strandberg TE, Tilvis RS: The impact of different diagnostic criteria on prevalence rates of delirium. Dement Geriatr Cogn Disord 2003;16:156-162.

-3 Inouye SK: Predisposing and precipitating factors for delirium in hospitalized older patients. Dement Geriatr Cogn Disord 1999;10:393-400.

-4 Laurila JV, Laakkonen ML, Strandberg TE, Tilvis RS, Pitkala KH: Predisposing and precipitating factors for delirium in a frail geriatric population. J Psychosom Res 2008;65:249-254.

-5 Inouye SK, Bogardus ST Jr, Charpentier PA, Leo-Summers L, Acampora D, Holford TR, Cooney LM Jr: A multicomponent intervention to prevent delirium in hospitalized older patients. N Engl J Med 1999;340:669-676.

-6 Pitkala KH, Laurila JV, Strandberg TE, Tilvis RS: Multicomponent geriatric intervention for elderly inpatients with delirium: a randomized, controlled trial. J Gerontol A Biol Sci Med Sci 2006;61:176181.

7 Lipowski ZJ: A new look at organic brain syndromes. Am J Psychiatry 1980;137:674-678.

8 Inouye SK: Delirium in older persons. N Engl J Med 2006;354:1157-1165.

-9 Flicker C, Serby M, Ferris SH: Scopolamine effects on memory, language, visuospatial praxis and psychomotor speed. Psychopharmacology (Berlin) 1990;100:243-250.

-10 Ancelin ML, Portet FS, Dupuy AM, Touchon J, Ritchie K: Non-degenerative mild cognitive impairment in elderly people and use of anticholinergic drugs: longitudinal cohort study. BMJ 2006;332: 455-459.

-11 Campbell N, Boustani M, Limbi T, Ott C, Fox C, Maidment I, Schubert CC, Munger S, Fick D, Miller D, Gulati R: The cognitive impact of anticholinergics: a clinical review. Clin Interv Aging 2009;4: $225-233$.

-12 Carrière I, Fourrier-Reglat A, Dartigues JF, Rouaud O, Pasquier F, Ritchie K, Ancelin ML: Drugs with anticholinergic properties, cognitive decline, and dementia in an elderly general population. The 3-city study. Intern Med 2009;169:1317-1324.

13 Boustani M, Campbell N, Munger S, Maidment I, Fox C: Impact of anticholinergics on the aging brain: a review and practical application. Aging Health 2008;4:311-320.

14 Flacker JM, Lipsitz LA: Neural mechanisms of delirium: current hypotheses and evolving concepts. J Gerontol 1999;54:239-246.

-15 Trzepacz PT: Is there a final common neural pathway in delirium? Focus on acetylcholine and dopamine. Semin Clin Neuropsychiatry 2000;5:132-148.

-16 Laurila JV, Pitkala KH, Strandberg TH, Tilvis RS: Delirium among patients with and without dementia: does the diagnosis according to the DSM-IV differ from the previous classifications? Int J Geriatr Psychiatry 2004;19:271-277.

- 17 Folstein MF, Folstein SE, McHugh PR: 'Mini-Mental State': practical method for grading the cognitive state of patients for the clinician. J Psychiatr Res 1975;12:189-198.

18 Wechsler D: The Measurement of Adult Intelligence. Baltimore, Williams \& Wilkins, 1944.

$\checkmark 19$ Hughes CP, Berg L, Danziger WL, Coben LA, Martin RL: A new clinical scale for the staging of dementia. Br J Psychiatry 1982;140:566-572.

20 Wechsler D: The Measurement and Appraisal of Adult Intelligence. Baltimore, Williams \& Wilkins, 1958.

21 American Psychiatric Association: Diagnostic and Statistical Manual of Mental Disorders, DSM-IV, ed 4. Washington, American Psychiatric Association, 1994.

-22 Charlson ME, Pompei P, Ales KL, MacKenzie CR: A new method of classifying prognostic comorbidity in longitudinal studies: development and evaluation. J Chronic Dis 1987;40:373-383.

23 Bazire S: Psychotropic Drug Directory 1996. The Professionals' Pocket Handbook \& Aide Memoire. London, Quay Books, 1995.

24 Brunton L, Lazo J, Parker K (eds): Goodman and Gilman's The Pharmacological Basis of Therapeutics, ed 11. New York, McGraw-Hill, 2005.

-25 Mintzer J, Burns A: Anticholinergic side-effects of drugs in elderly people. J R Soc Med 2000;93: $457-462$. 
-26 Chutka DS, Takahashi PY, Hoel RW: Inappropriate medications for elderly patients. Mayo Clin Proc 2004;79:122-139.

-27 Uusvaara J, Pitkala KH, Tienari PJ, Kautiainen H, Tilvis RS, Strandberg TE: Association between anticholinergic drugs and apolipoprotein E4 allele and poorer cognitive function in older cardiovascular patients: a cross-sectional study. J Am Geriatr Soc 2009;57:427-431.

-28 Fick DM, Cooper JW, Wade WE, Waller JL, Maclean JR, Beers MH: Updating the Beers criteria for potentially inappropriate medication use in older adults: results of a US consensus panel of experts. Arch Intern Med 2003;163:2716-2724.

-29 Panula J, Puustinen J, Jaatinen P, Vahlberg T, Aarnio P, Kivela SL: Effects of potent anticholinergics, sedatives and antipsychotics on postoperative mortality in elderly patients with hip fracture: a retrospective, population-based study. Drugs Aging 2009;26:963-971.

-30 Tune L, Carr S, Hoag E, Cooper T: Anticholinergic effects of drugs commonly prescribed for the elderly: potential means for assessing risk of delirium. Am J Psychiatry 1992;149:1393-1394.

- 31 Rudolph JL, Salow MJ, Angelini MC, McGlinchey RE: The anticholinergic risk scale and anticholinergic adverse effects in older persons. Arch Intern Med 2008;168:508-513. 